\title{
Ammoniac et poissons lors de la vidange d'une retenue
}

\section{Ammonia and fish during the draining of a reservoir}

\author{
P. Francisco*, D. Escorihuela*, J.N. Tourenq ${ }^{*}$ et J.P. Parent ${ }^{\star}$
}

* Centre d'Ecologie des Systemes Aquatiques Continentaux - UMR C 5576 CNRS/ Université Paul Sabatier, 118 route de Narbonne, F-31062 Toulouse Cedex 4, France.

Résumé. - Des concentrations proches de $0,3 \mathrm{mg} \cdot \mathrm{f}^{-1}$ d'ammoniac non ionisé $\left(\mathrm{NH}_{3}\right)$ relevées dans l'épilimnion ainsi que des températures de $22^{\circ} \mathrm{C}$ et des $\mathrm{pH}$ voisins de 11 , nous ont conduit à suivre l'évolution de ces paramètres au cours de la vidange de la retenue de Pont de Salars (Aveyron). L'absence de mortalité et le bon état sanitaire des poissons récupérés en fin de vidange laissent supposer une hétérogénéité physico-chimique de la retenue dont la communauté pisciaire pourrait tirer parti évitant ainsi une exposition prolongée à ces fortes concentrations en $\mathrm{NH}_{3}$.

Mots clés. - Ammoniac, poissons, retenue, vidange.

Abstract. - The presence in the epilimnion of un-ionized ammonia ( $\mathrm{NH} 3$ ) concentrations close to $0.3 \mathrm{mg} . \mathrm{l}^{-1}$, with water temperatures of $22^{\circ} \mathrm{C}$ and $\mathrm{pH}$ values around 11 , led us to monitor the evolution in these parameters during draining of the Pont de Salars reservoir (Aveyron). The absence of fish mortality and the good condition of fish collected at the end of the draining are indications of the physico-chemical heterogeneity in the reservoir. This should be beneficial to the fish in that it tends to prevent prolonged exposure to high $\mathrm{NH}_{3}$ concentrations.

\section{INTRODUCTION}

En solution aqueuse, l'ammoniaque se présente sous deux formes chimiquement distinctes. La forme non ionisée $\left(\mathrm{NH}_{3}\right)$ est en équilibre avec l'ion ammonium $\left(\mathrm{NH}_{4}^{+}\right)$et l'ion hydroxyde. La proportion relative de ces deux formes dépend en particu- lier du $\mathrm{pH}$ et de la température de l'eau (Trussel, 1972; Emerson et al., 1975). La toxicité de l'ammoniaque $\left(\mathrm{NH}_{4} \mathrm{OH}\right)$ vis à vis des organismes aquatiques est essentiellement due à la forme non ionisée (ANI) (Wuhrmann et Woker, 1948 ; Lloyd, 1961). Les seuils de toxicité ainsi que les effets de l'ammoniac ont été gé- 
néralement étudiés chez des poissons maintenus en milieu contrôlé (Fromm et Gillette, 1968; Lloyd et Orr, 1969 ; Smart, 1976 ; Alabaster et Lloyd, 1980 ; Jeney et al., 1992).

Lors d'un contrôle des paramètres physico-chimiques de l'eau réalisé avant la vidange de la retenue de Pont de Salars (Aveyron), il a été noté, en surface, de fortes concentrations en ammoniac ainsi que des valeurs de $\mathrm{pH}$ proches de 11.

Aussi, il a paru intéressant de suivre, les variations éventuelles des concentrations élevées en $\mathrm{NH}_{3}$ et leurs effets possibles sur la population pisciaire de la retenue au cours de la vidange.

\section{SITE, MATÉRIEL ET MÉTHODES}

Situé sur le plateau du Lévézou (Aveyron, France), à une altitude de 718 mètres, le lac de Pont de Salars fait partie de l'aménagement hydroélectrique du Pouget; alimenté par le Viaur, il fonctionne en vases communiquants avec le réservoir de Bage par l'intermédiaire d'une galerie de jonction.

La retenue de Pont de Salars appartient à la catégorie des lacs monomictiques chauds avec une nette stratification de mai à octobre; elle est généralement qualifiée de mésotrophe avec une tendance à l'eutrophisation durant la période estivale. Les caractéristiques morphométriques sont indiquées dans la figure 1 .

La vidange a débuté le 21 août 1995 et s'est poursuivie jusqu'au $1^{\text {er }}$ septembre 1995. L'eau a été soutirée d'une part par l'intermédiaire de la galerie de Bage (cote 702,6-705 m NGF) avec un débit moyen d'environ $10 \mathrm{~m}^{3} \cdot \mathrm{s}^{-1}$ et d'autre part grâce à l'ouverture des vannes de fond (cote $686,4 \mathrm{~m} \mathrm{NGF}$ ) pour un débit équivalent.

La température, l'oxygène dissous, le $\mathrm{pH}, \mathrm{NH}_{4}-\mathrm{N}$ et $\mathrm{NH}_{3}$ ont été suivis du 20 au 29 août 1995.

Les profils verticaux de température et d'oxygène dissous ont été réalisés à l'aide d'une sonde (YSI) deux fois par jour ( $8 \mathrm{~h} 00$ et $18 \mathrm{~h} 00$ ) en un point situé à proximité des deux prises d'eau et pouvant être considéré comme représentatif de la colonne d'eau immédiatement en amont du barrage. Simultanément, des prélèvements d'eau ont été effectués à l'aide d'une bouteille de type Van Dorn (5 litres) depuis la surface jusqu'au fond. Pour chaque prélèvements, des mesures de $\mathrm{pH}$, d'oxygène dissous (méthode de Winkler) et des dosages d'azote ammoniacal (méthode de Nessler) ont été réalisés in situ. Le pourcentage de la fraction non ionisé ANI $\left(\mathrm{NH}_{3}\right)$ de l'ammoniac total a été calculé à l'aide de la relation:

$\left.\% \mathrm{NH}_{3}(\mathrm{ANI})=100 / 1+10^{(10,058-(0,0316 \mathrm{~T}) \text {-pH })}\right]$.

Malgré une tendance à l'eutrophisation de la retenue en période estivale, les valeurs des paramètres mesurés au cours de notre étude ne 


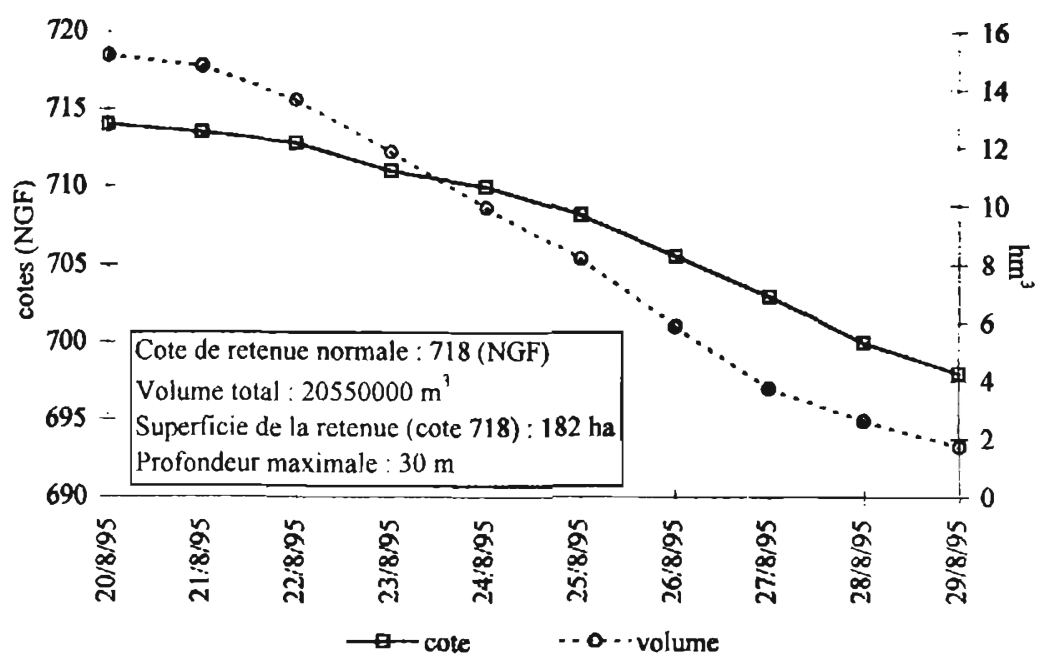

Fig. 1. - Variation de la cote et du volume de la retenue au cours de la vidange.

Fig. 1. - Variation in water level and volume of the reservoir during the draining.

montrent aucune différence significatives entre les deux prélèvements quotidiens ( $8 \mathrm{~h} 00$ et $18 \mathrm{~h} 00$ ). De ce fait, des moyennes journalières ont été calculées et sont présentées dans la figure 2.

En raison de l'inaccessibilité du plan d'eau, aucun prélèvement n'a pu être conduit au cours des trois derniers jours de la vidange (30 août $1^{\text {er }}$ septembre).

La biomasse pisciaire de la retenue a été évaluée après récupération complète des animaux à la pêcherie située immédiatement à l'aval du barrage puis les animaux ont été répartis dans différents plans d'eau avoisinants.

\section{RÉSULTATS}

Les profils verticaux enregistrés le 20 août 1995 rendent compte de l'état physico-chimique du lac avant le début de la vidange (fig. 2.). On note une stratification thermique bien marquée où les températures épilimniques avoisinent les $23^{\circ} \mathrm{C}$ puis chutent rapidement entre 7,5 et 10 mètres de profondeur au niveau de la thermocline pour atteindre des valeurs de l'ordre de $8^{\circ} \mathrm{C}$ dans l'hypolimnion. Les concentrations en oxygène dissous évoluent de façon similaire donnant un épilimnion sursaturé $\left(13 \mathrm{mg} . \mathrm{I}^{-1}\right)$ et un hypolimnion caractérisé par de faibles valeurs $\left(3 \mathrm{mg} . \mathrm{I}^{-1}\right)$. Le $\mathrm{pH}$ est élevé dans la strate 0-5 mètres de 


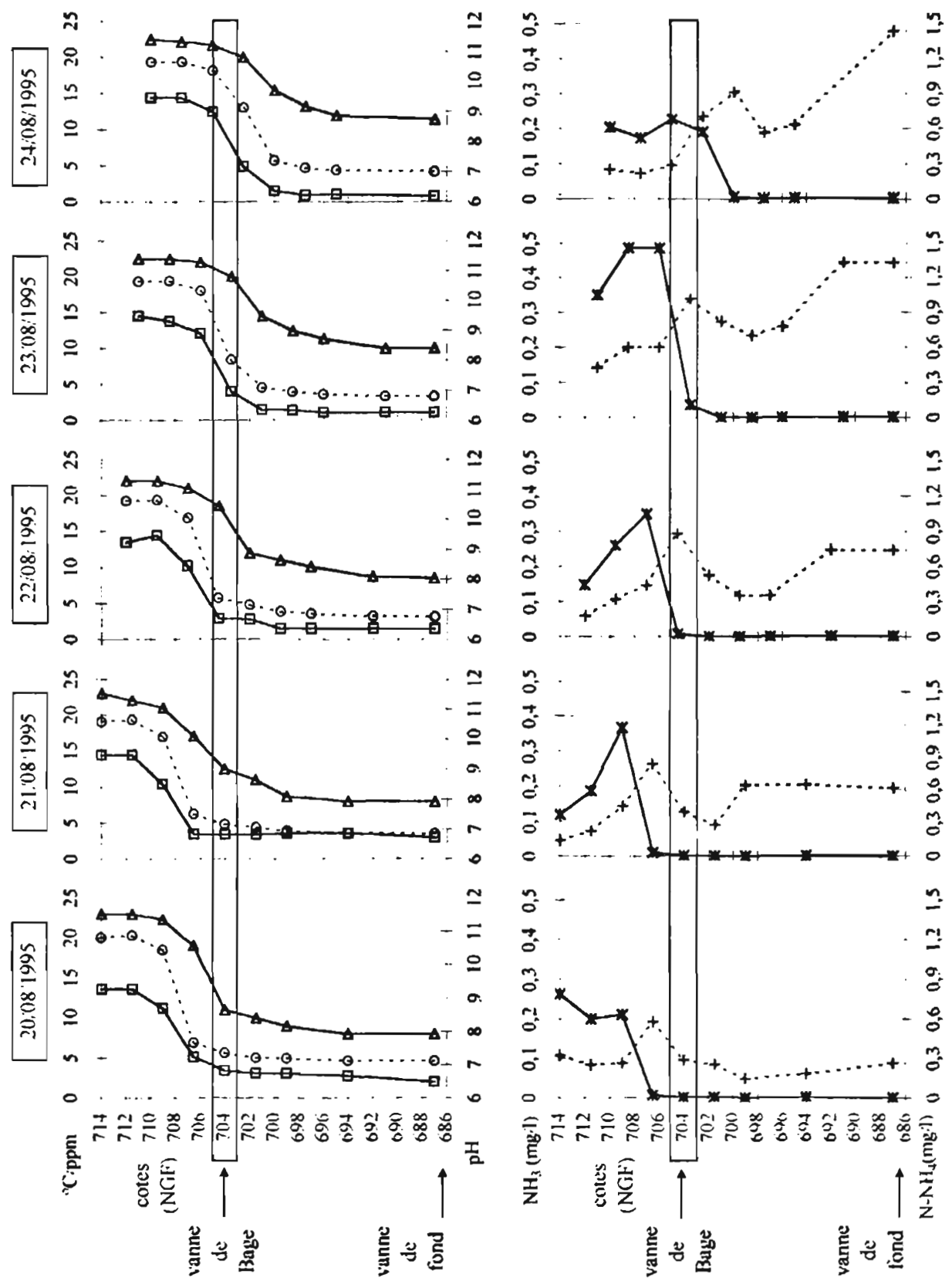

Fig. 2. - Evolution des profils verticaux de température (- - ), d'oxygene dissous (-7-), de pH (-- )--). $\mathrm{N}^{-\mathrm{NH}_{4}(--+-)}$ et $\mathrm{NH}_{3}(-x-)$ au cours de la vidange.

Fig. 2. - Evolution of the vertical profiles of temperature (-, ), dissolved oxygen (-7), pH (- )- ), $\mathrm{N}-\mathrm{NH}_{4}(--+-)$ and $\mathrm{NH}_{3}(-x-)$ during the draining. 


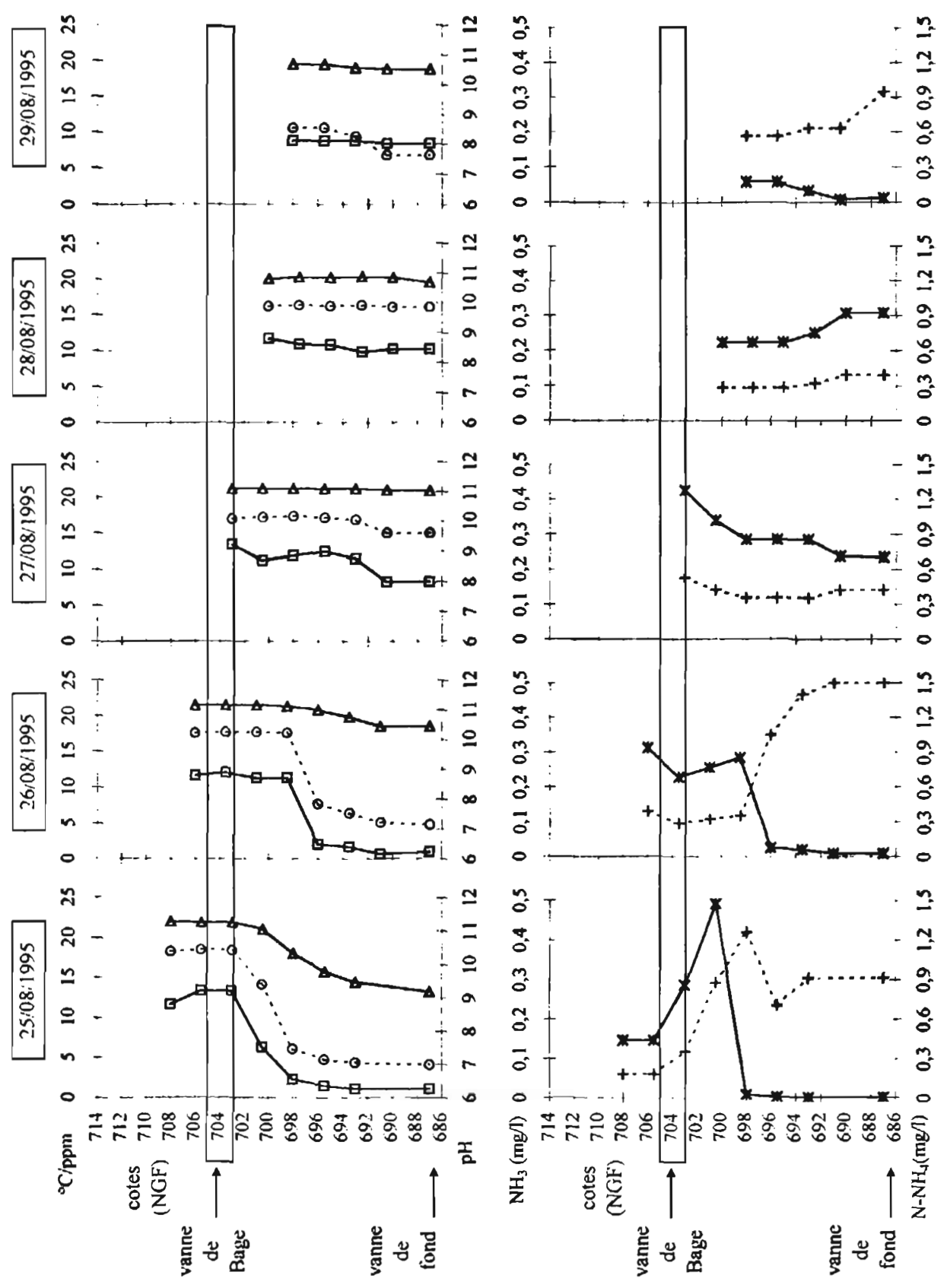


profondeur $(10,8)$ et se stabilise aux alentours de la neutralité dans l'hypolimnion. L'ensemble de la colonne d'eau est caractérisé par des concentrations en $\mathrm{NH}_{4}-\mathrm{N}$ oscillant entre 0,2 et $0,3 \mathrm{mg} \cdot \mathrm{l}^{-1}$. On observe toutefois un maximum de $0,58 \mathrm{mg}^{-\mathrm{l}^{-1}}$ à 7,5 mètres de profondeur. Les concentrations en $\mathrm{NH}_{3}$ sont supérieures à $0,2 \mathrm{mg} . \mathrm{l}^{-1}$ dans la strate 0-5 mètres. En profondeur, les valeurs enregistrées sont proches de 0 .

Durant toute la première partie de la vidange (21-25 août), le soutirage de l'eau au niveau de la thermocline puis de l'épilimnion par l'intermédiaire de la galerie de Bage, n'a pas entraîné de modification de la stratification en place. Les profils de température, d'oxygène dissous et de $\mathrm{pH}$ sont identiques à ceux précédemment observés.

Les valeurs moyennes des concentrations en ANI calculées sur la strate 0-5 mètres restent supérieures à 0.2 mg. $\mathrm{l}^{-1}$, un maximum de $0,5 \mathrm{mg} . \mathrm{l}^{-1}$ est relevé le 25 août à 7,5 mètres. Au niveau de la thermocline, il faut noter l'existence d'une zone dans laquelle les teneurs en ANI sont inférieures à $0,02 \mathrm{mg} \cdot \mathrm{l}^{-1}$ et où celles en oxygène dissous sont de l'ordre de $5 \mathrm{mg}^{-1}$. En profondeur, les températures et $\mathrm{pH}$ enregistrés n'entraînent pas de fortes concentrations en $\mathrm{ANI}$ et ce malgré des valeurs parfois élevées en ammoniac total $\left(1,4 \mathrm{mg}^{-1}{ }^{-1}\right.$ le 24 août à 22 mètres).

Au cours de cette phase d'abaissement du plan d'eau, seule l'épais- seur de la tranche d'eau hypolimnique a progressivement diminuée.

Le 26 août, la hauteur de l'hypolimnion ne représente plus que $50 \%$ de la colonne d'eau totale. La surface de la retenue est proche du niveau supérieur de la vanne de Bage, le volume restant $\left(6 \mathrm{hm}^{3}\right.$, fig. 1) est constitué à près de $80 \%$ par l'épilimnion. On observe alors le début de la disparition de la stratification thermique. Les différences de températures relevées sur la colonne d'eau n'excèdent pas $3^{\circ} \mathrm{C}$. En revanche, les gradients d'oxygène dissous, de $\mathrm{pH}$ et d'ANI sont maintenus.

A partir du 27 août, la prise d'eau de la galerie de Bage n'est plus fonctionnelle. Durant la seconde partie de la vidange, l'eau est désormais évacuée uniquement par la vanne de fond.

Les profils révèlent alors une homogénéisation complète de la masse d'eau. La retenue est caractérisée par des concentrations en oxygène dissous, des valeurs de $\mathrm{pH}$ et de température correspondant à celles relevées précédemment dans l'épilimnion. L'élévation du $\mathrm{pH}$ dans la zone profonde entraîne à ce niveau une hausse de I'ANI. La concentration moyenne en ANI sur l'ensemble de la colonne d'eau est d'environ $0,3 \mathrm{mg} . \mathrm{l}^{-1}$.

Les jours suivant, la masse d'eau reste homogène. Hormis l'oxygène, les paramètres mesurés tendent à diminuer. La température moyenne sur la colonne d'eau est de $19^{\circ} \mathrm{C}$, le $\mathrm{pH}$ 
de 8 et la concentration en $\mathrm{NH}_{3}$ est proche de 0,03 mg. $\mathrm{I}^{-1}$.

Durant toute la période stratifiée, aucun poisson n'a été observé à la pêcherie. Après l'homogénéisation de la masse d'eau (28 août), quelques individus isolés (perches, sandres) ont été récupérés à l'aval du barrage. L'essentiel de la récupération piscicole s'est opéré durant les nuits du 29 au 30 août et du 30 août au $1^{\text {er }}$ septembre 1995.

La biomasse pisciaire est d'environ 35 tonnes. Le gardon (Rutilus rutilus, L.) représente $67,5 \%$ de la biomasse totale, le sandre (Stizostedion lucioperca, L.) $12 \%$, la tanche (Tinca tinca, L.) $7 \%$, la carpe (Cyprinus carpio, L.) $6,4 \%$, le brochet (Esox lucius, L.) $3 \%$, la perche (Perca fluviatilis, L.) $3 \%$, le chevesne (Leuciscus cephalus, L.) $1 \%$ et les divers (ablettes, goujons et truites) $0,1 \%$.

\section{DISCUSSION}

La répartition verticale des paramètres physico-chimiques étudiés est en accord avec les profils observés dans des milieux lacustres eutrophes (Wetzel 1983 ; Dussart 1992).

Durant la période de stratification, les fortes teneurs en oxygène dissous de l'épilimnion révèlent une activité photosynthétique élevée de la population algale entraînant une consommation du $\mathrm{CO}_{2}$ suivie en général d'une élévation du $\mathrm{pH}$. Inversement, la chute des concentrations en oxygène dissous en profondeur, témoi- gne de son utilisation lors des processus de dégradation de la matière organique. Simultanément, l'augmentation du $\mathrm{CO}_{2}$ dans l'hypolimnion résultant de ces processus tend à diminuer le $\mathrm{pH}$. L'accumulation de $\mathrm{NH}_{4}-\mathrm{N}$ en profondeur pendant la stratification confirme la forte productivité de ce réservoir.

Alors que la stratification thermique disparaît (26-27 août), le lac est caractérisé en profondeur par une forte diminution des concentrations en $\mathrm{NH}_{4}-\mathrm{N}$ qui pourrait être due à l'évacuation massive de l'eau hypolimnique (chargé en $\mathrm{NH}_{4}-\mathrm{N}$ ) par les vannes de fond.

Après l'homogénéisation complète (27 août) et en raison de l'importance relative du volume épilimnique, le $\mathrm{pH}$ et les concentrations en ANI restent élevés $\left(0,3 \mathrm{mg.}^{-1}\right.$ en moyenne) durant les 48 heures suivantes. En fin de vidange, la diminution progressive de ces deux paramètres pourrait être expliquée par les apports du Viaur caractérisé par une eau plus froide et dont le $\mathrm{pH}$ est voisin de la neutralité.

La demande en oxygène dissous des poissons dépend de l'espèce considérée, de son stade de développement (œuis, larves ou adultes) ou encore du statut physiologique auquel l'on se réfère (croissance, reproduction). II est donc difficile de définir, pour un milieu donné, la zone de dépendance respiratoire en deçà de laquelle la population pisciaire peut être menacée. Toutefois, on peut considérer que des concentrations de $3{\mathrm{mg} . \mathrm{I}^{-1}}^{-1}$ 
constituent le minimum requit pour la survie des poissons (Alabaster et Lloyd, 1980). Le déficit hypolimnique en oxygène observé dans la colonne d'eau située immédiatement à l'amont du barrage implique que l'essentiel de la communauté pisciaire soit réparti soit dans les strates superficielles soit dans des zones où la concentration en oxygène dissous est suffisante pour la survie des animaux (e.g. au niveau de la thermocline, dans d'autres parties plus amont dans la retenue).

Les valeurs de $\mathrm{pH}$ supérieures à 10 relevées dans l'épilimnion représentent, pour une courte période d'exposition, le maximum tolérable par le gardon. La carpe, la tanche et le brochet, espèces plus résistantes à l'alcalinisation, ne survivent cependant pas à une exposition prolongée pour des $\mathrm{pH}$ compris entre 10,5 et 11 (Alabaster et Lloyd, 1980). De plus, outre les effets directs de l'élévation du $\mathrm{pH}$, il faut ajouter l'action toxique de l'ammoniac. Le pourcentage d'ammoniac non ionisé est multiplié par 10 lorsque l'on passe d'un $\mathrm{pH}$ de 8 à un pH de 9 (Emerson et al., 1975).

La toxicité de l'ammoniac a été largement étudiée chez les Salmonidae (Lloyd, 1961; Fromm et Gillette, 1968 ; Lloyd et Orr, 1969; Rice et Stokes, 1975; Thruston et al., $1984 ; \ldots$ ). Considérés comme les plus sensibles à la présence d'ammoniac, le seuil de toxicité préconisé en pis-

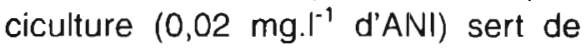
référence pour l'ensemble des pois- sons. Cependant, Schuze-wiehenbranck (1976) montre que des con-

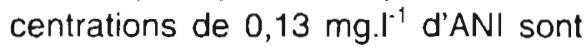
sans effet sur la croissance de la truite arc-en-ciel (Oncorhynchus mykiss) et que seules celles supérieures à $0,15 \mathrm{mg} . \mathrm{I}^{-1}$ affectent temporairement la croissance des animaux. Forster et Smart (1979) rapportent que des concentrations de $0,1{\mathrm{mg} . l^{-1}}^{-1}$ provoquent une léthargie, une perte de l'appétit et une diminution de la croissance seulement durant les deux premières semaines de l'exposition. Les poissons semblent ensuite s'acclimater à ces valeurs et la croissance est à nouveau normale. Les rapports de I'EIFAC (1970) et de l'US Environmental Protection Agency (1976) proposent respectivement des concentrations de 0,021 et $0,016 \mathrm{mg}^{-1}$ d'ANI comme étant la limite supérieure tolérable par les poissons sur de longues périodes.

La détermination des doses sublétales et létales de l'ammoniac non ionisé dépend de l'espèce et de la durée d'exposition. Dans un récapitulatif des travaux existant, Alabaster et Lloyd (1980) rapportent que la $\mathrm{CL}_{50}$ $96 \mathrm{~h}$ est de $0,35 \mathrm{mg} . \mathrm{l}^{-1}$ pour la perche et de $0,42 \mathrm{mg}^{-\mathrm{I}^{-1}}$ pour le gardon. D'autres espèces telles que la carpe et la tanche sont plus résistantes aux fortes concentrations en $\mathrm{NH}_{3}$ en particulier pour de brèves durées d'exposition (jusqu'à $2 \mathrm{mg} \cdot \mathrm{l}^{-1}$ d'ANI). Dabrowska et Sikora (1986) montrent, chez des carpes âgées de 1 an, que la dose létale est comprise entre 1,15 et $1,96 \mathrm{mg}^{-1}$. 
Lors de la récupération des poissons, l'examen macroscopique de plusieurs individus sacrifiés pour chaque espèce n'a révélé aucun symptôme attestant d'un état sanitaire précaire des animaux (hydroplisie, hypersécrétion de mucus, lésion hépatique ou vasculaire...). La présence de nombreuses proies dans les contenus stomacaux ou intestinaux examinés, montre qu'il n'y a pas eu de diminution importante de la prise alimentaire pendant la phase d'abaissement du plan d'eau. et semble indiquer que les animaux n'auraient pas subit une exposition prolongée à de mauvaises conditions environnementales dues en particulier aux fortes concentrations en $\mathrm{NH}_{3}$ mesurées en amont du barrage au cours de la vidange de cette retenue.

\section{CONCLUSION}

Dans la retenue de Pont de Salars, immédiatement en amont du barrage, les conditions physico-chimiques relevées dans la colonne d'eau ne sont pas favorables à la communauté pisciaire (fortes concentrations en ANI, $\mathrm{pH}$ élevé, faible oxygénation) et ce, jusqu'à l'homogénéisation complète des masses d'eaux (le 28 août). Ceci pourrait expliquer l'absence de passage des poissons au niveau des vannes de fond pendant toute cette période. En effet, c'est seulement lorsque les conditions du milieu sont devenues moins défavorables (diminution des concentrations en ANI, abaissement du $\mathrm{pH}$ et meilleure oxy- génation) que s'est opéré le passage des animaux (à partir du 29 août). La détermination et le comptage des poissons réalisée à la pêcherie ont permis de dégager, en fonction du temps, trois phases successives de passage des espèces (sandre, perche (perche, brochet (tanche, carpe, gardon), chronologie qui reflète probablement la résistance croissante de ces espèces aux conditions de milieu difficiles, les poissons les plus robustes dévalant en fin de vidange.

Cependant, ce travail laisse en suspend la question de l'applicabilité au milieu naturel des normes de qualité d'eau pour le $\mathrm{NH}_{3}$ issues de tests toxicologiques, mais peut suggérer l'existence de "zones de refuges", conséquence d'une hétérogénéité du milieu, dans lesquelles les poissons ne seraient plus soumis à des conditions environnementales peu propices à leur développement ou à leur survie.

Pour préciser, dans un lac de retenue, les valeurs limites de tolérance des poissons aux concentrations en ANI, il serait nécessaire de localiser par échosondage les populations pisciaires et d'effectuer simultanément un plus grand nombre de points de mesures de la qualité physico-chimique de l'eau.

\section{RÉFÉRENCES}

Alabaster (J.S.) \& Lloyd (R.) 1980. Water quality criteria for freshwater fish. London Butterworths : $297 \mathrm{pp}$. 
Dabrowska (H.) \& Sikora (H.) 1986. Acute toxicity of ammonia to common carp (Cyprinus carpio, L.). Pol. Arch. Hydrobiol., 33 : 121-128.

Dussart (B.) 1992. Limnologie - L'étude des eaux continentales $2^{\text {nd }}$ édition. Ed. Boubée et $\mathrm{C}^{\mathrm{ie}}$ : $680 \mathrm{pp}$.

EIFAC 1970. Water quality criteria for European freshwater fish. Report on ammonia and inland fisheries. EIFAC Tech. Pap., 11: 12 pp.

Emerson (K), Russo (R.C.), Lund (R.E.) \& Thurston (R.V.) 1975. Aqueous ammonia equilibrium calculations : effect of $\mathrm{pH}$ and temperature. J. Fish. Res. Bd. Canada., 32 : 2379-2383.

Forster (J.R.) \& Smart (G.R.) 1979. Water economy in aquaculture. Power Plant Waste heat Utilisation in Aquaculture. Godfriaux (B.L.), Able (A.S.), Farmanarmaian (A.), Greene (C.R.) \& Stevens (C.A.) eds. New Jersey, USA: Allanheld, Osman and Co. : 3-11.

Fromm (P.O.) \& Gillette (J.R.) 1968. Effect of ambient ammonia on blood ammonia and nitrogen excretion of rainbow trout (Salmo gairdnerii). Comp. Biochem. Physiol., 26 : 887-896.

Jeney (Z.), Nemcsok (J.), Jeney (G.) \& Olah (J.) 1992. Acute effect of sublethal ammonia concentrations on common carp (Cyprinus carpio, L.) : Effect of ammonia on adrenaline and noradrenaline levels in different organs. Aquaculture., 104 (1-2) : 139148.

Lloyd (R.) 1961. The toxicity of ammonia to rainbow trout (Salmo gairdnerii Richardson). Water Waste Treat., 8: 278-281.

Lloyd (R.) \& Orr (L.D.) 1969. The diuretic response by rainbow trout to sub- lethal concentrations of ammonia. Water Res., 3 : 335-344.

Rice (S.D.) \& Stokes (R.M.) 1975. Acute toxicity of ammonia to several developmental stages of rainbow trout (Salmo gairdnerii). Fish Bull., 73 : 207 211.

Smart (G.) 1976. The effect of ammonia exposure on gill structure of the rainbow trout (Salmo gairdnerii). J. Fish. Biol., $8: 471-475$.

Schuze-Wiehenbranck $(H$.) 1976. Effect of sublethal ammonia concentrations on metabolism in juvenil rainbow trout (Salmo gairdnerii Richardson). Ber. Dt. Wiss. Kommn. Meeresforsch., 24 : 234-250.

Thurston (R.V.), Russo (R.C.), Luedtke (R.J.), Smith (C.E.), Meyn (E.L.), Chacoumakos (C.), Wang (K.C.) \& Brown (C.D.J.) 1984. Chronic toxicity of ammonia to rainbow trout. Trans. Am. Fish. Soc., 113 (1) : 56-73.

Trussel (R.P.) 1972. The percent un-ionized ammonia in aqueous ammonia solutions at different $\mathrm{pH}$ levels and temperatures. J. Fish. Res. Bd. Canada., $29:$ 1505-1507.

U.S. Environmental Protection Agency 1976. Quality criteria for water. Washington D.C. : U.S. Government Printing Office. : $256 \mathrm{pp}$.

Wetzel (R.) 1983. Limnology $2^{\text {nd }}$ edition. Saunders College Publishing : 767 pp.

Wurhmann (K.von) \& Woker (H.) 1948. Beitrage zur toxikologie der fishe - II Experimetelle untersuchungen über die ammoniak-und blausäurevergiftung. Schweiz. Z. Hydrol., 11: 210244. 